\title{
Appendix Undifferentiated Carcinoma
}

National Cancer Institute

\section{Source}

National Cancer Institute. Appendix Undifferentiated Carcinoma. NCI Thesaurus. Code C43556.

A high grade carcinoma arising from the appendix, characterized by the absence of glandular or squamous differentiation. 